\title{
Patterns of Spinal Cord Atrophy by Metrizamide CT
}

\author{
Michel E. Mawad, ${ }^{1}$ Sadek K. Hilal, ${ }^{1}$ Michael R. Fetell, ${ }^{2}$ A. John Silver, ${ }^{1}$ S. Ramaiah Ganti, ${ }^{1}$ and Paul Sane ${ }^{1}$
}

Forty patients with cervical myelopathy underwent high-resolution computed tomography (CT) with intrathecal administration of metrizamide for evaluation of cervical spinal cord atrophy. Thirty of them showed evidence of either focal atrophic distortion or generalized accentuation of the anatomic surface features of the spinal cord. Patients with a Chiari malformation or syringomyelia were excluded. The characteristic features in cervical spondylosis and canal deformity include flattening of the ventral surface of the cord, central infolding, beaking of the lateral funiculi, and wasting of the dorsal surface of the cord. Patients with motor neuron disease showed a combination of anterolateral and posterolateral atrophy reflecting underlying degeneration of the anterior horn cells and/or corticospinal tracts, respectively. Those with monomelic motor neuron disease had a striking ipsilateral hemiatrophy of the spinal cord. Among those presenting with spastic paraparesis, seven with clinically definite multiple sclerosis showed diffuse atrophy or focal degeneration due to a localized plaque of demyelination. Two cases of cord neoplasm showed atrophy secondary to ascending or descending degeneration of the long tracts.

High-resolution computed tomography (CT) with intrathecal metrizamide as a contrast agent has become an increasingly reliable tool in the diagnosis of spinal cord disease. While enlargement of the spinal cord secondary to a neoplasm or syrinx is readily recognizable on iophendylate or air myelography, conventional myelography is misleading in cases of spinal cord atrophy. Routine myelographic imaging relies on two perpendicular projections of the spinal canal and its contents, while metrizamide CT produces a clear, nonsuperimposed cross-sectional image of the bony canal, the dural sac opacified with metrizamide, and the spinal cord. Metrizamide opacifies the subarachnoid space and fills the fissures and sulci on the surface of the cord, enabling early detection of subtle anatomic deformity and distortion. It is possible with metrizamide CT to detect cord atrophy with preservation of one or both of the sagittal and transverse diameters. We have found it an excellent method for studying subtle deformities of the spinal cord in vivo without the interference of fixation artifacts such as are encountered in postmortem studies.

\section{Subjects and Methods}

Forty patients with cervical myelopathy were studied with metrizamide CT at our institution during an 18 month period. Fourteen of them had spondylotic myelopathy with myelographic evidence of cord compression or spinal canal deformity. Eleven patients had upper or lower motor neuron disease diagnosed clinically and confirmed by electromyography. Eleven presented with spastic paraparesis or quadriparesis; seven of these had clinically definite multiple sclerosis with oligoclonal bands in the cerebrospinal fluid, while the other four had probable multiple sclerosis. The series also included two cases of cord degeneration rostral and caudal to a tumor, one case of biopsy-proven cord atrophy of unknown etiology, and one case of postsurgical atrophy.

Scanning was performed on a 0450 Pfizer machine after lumbar subarachnoid injection of $10-12 \mathrm{ml}$ metrizamide $(170 \mathrm{mg} / \mathrm{ml}$ iodine). The area scanned extended from the $\mathrm{T} 1$ level through the midbrain. Slice thickness and spacing was $5 \mathrm{~mm}$. Hospital records including laboratory, electromyographic, and evoked-potential workups of all patients were carefully reviewed by one of the authors (M. R. F.). A more detailed correlation of the clinical and radiographic findings will be the subject of a future report.

\section{Results}

The clinical findings and radiographic observations are subdivided into three major groups. Several other cases of cord atrophy of various etiologies are listed separately.

\section{Cervical Spondylosis}

All patients in this group had a minimal sagittal diameter of the spinal canal of $15 \mathrm{~mm}$ or less. The early pressure deformity produced by an osteophyte is flattening of the ventral surface of the cord; this progresses to central infolding and a bean-shaped cord with widening of the ventral fissure (fig. 1A). The dentate ligament maintains a tension effect on the lateral funiculi which, under pressure from the osteophyte anteriorly, become thin and beaked, pointing toward the anterolateral recesses of the spinal canal (fig. $1 \mathrm{~B}$ ). With further narrowing of the spinal canal, the anterolateral and posterolateral funiculi of the cord become atrophic. Those patients with sensory changes and asterognosis of the hands had striking dorsal column atrophy. (fig. 1C). None of these findings were encountered in patients undergoing metrizamide CT who did not have cervical myelopathy.

\section{Motor Neuron Disease}

Patients with monomelic lower motor neuron disease showed ipsilateral flattening of the anterolateral funiculus of the spinal cord

'Department of Radiology, Neurological Institute, Columbia-Presbyterian Medical Center, 710 W. 168th St., New York, NY 10032 . Address reprint requests to M. E. Mawad.

${ }^{2}$ Department of Neurology, Neurological Institute, Columbia-Presbyterian Medical Center, New York, NY 10032.

AJNR 4:611-613, May/June 1983 0195-6108/83/0403-0611 \$00.00 (C) American Roentgen Ray Society 


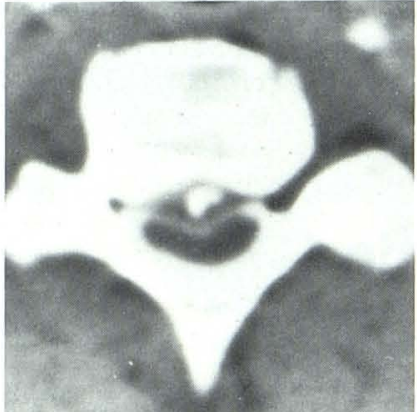

A

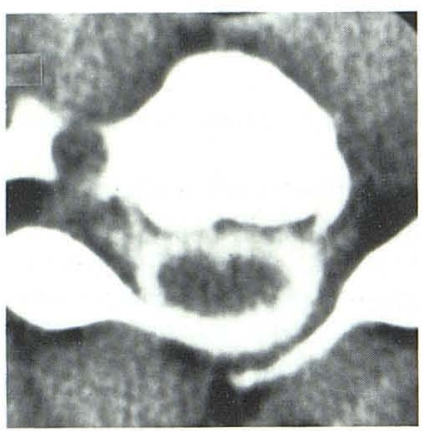

A

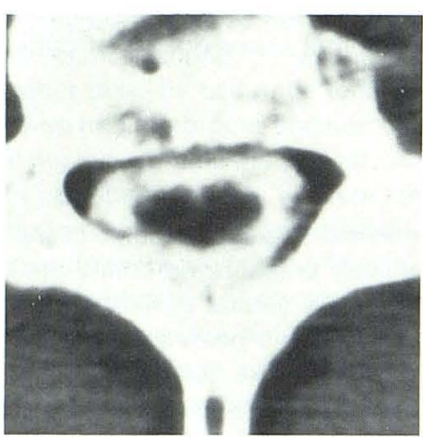

A

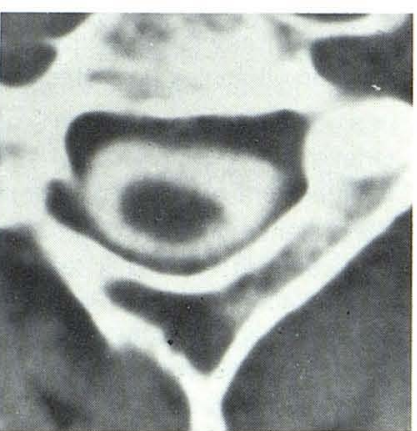

B

Fig. 3.-Multiple sclerosis. A, Nonspecific generalized atrophy of spinal cord with widening of ventral fissures and accentuation of surface sulci. B, Ipsilateral focal hemiatrophy of cervical cord in patient with left-sided hyperpathia from $\mathrm{C} 2$ to $\mathrm{T} 2$, probably due to demyelinating plaque.

without widening of the ventral fissure (fig. 2A). Patients with amyotrophic lateral sclerosis had flattening of both the anterolateral and posterolateral funiculi (fig. 2B). The ventral fissure was preserved. The brainstem in these patients often showed pyramidal atrophy in the pons and medulla (fig. 2C).

\section{Spastic Paraparesis}

Within this group, seven patients with definite clinical evidence of multiple sclerosis had nonspecific atrophy of the spinal cord

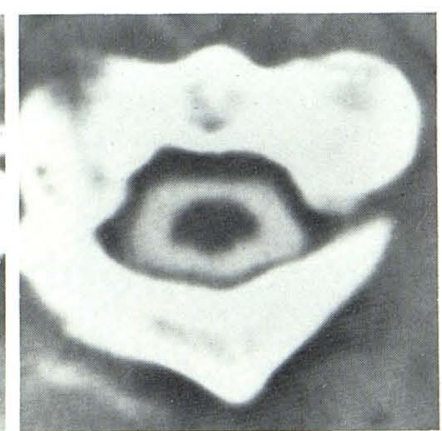

Fig. 1.-Cervical spondylosis. A, Central infolding of spinal cord with widening of ventral fissure produced by direct pressure from osteophyte. B, Beaking of lateral funiculi, which taper under pressure and point toward anterolateral recesses of spinal canal. C, "Notching" deformity of dorsal surface of cord due to cuneate atrophy in patient with astereognosis of hands.
Fig. 2.-Motor neuron disease. A, Ipsilateral hemiatrophy of spinal cord in patient with monomelic lower motor neuron disease. B, Amyotrophic lateral sclerosis. Flattening of both anterior and posterior surfaces of cord due to underlying degeneration of anterior horn cells and corticospinal tracts. C, Pyramidal atrophy of medulla, also seen in amyotrophic lateral sclerosis. including widening of the ventral fissure and exaggeration of the surface sulci (fig 3A). There was no evidence of cord infolding or beaking of the lateral funiculi as seen in spondylosis. Three patients had evidence of brainstem atrophy. One patient with a long history of left arm weakness and hyperpathia had ipsilateral cord hemiatrophy (fig. 3B).

\section{Other Cervical Myelopathies}

One patient with intramedullary astrocytoma and another with a foramen magnum extraaxial tumor had, respectively, ascending degeneration with widening of the ventral fissure and descending degeneration with hemiatrophy. The most striking case of cord atrophy was seen in a patient with a long-standing undiagnosed myelopathy who had undergone multiple decompressive laminectomies without relief. His cord biopsy showed primary atrophy with no evidence of demyelination or motor neuron degeneration. In another case involving an intramedullary nodule of hemangioblastoma and a wide cord, postoperative CT showed diffuse nonspecific atrophy of the cord with widening of the ventral fissure.

\section{Discussion}

The clinical aspects and pathogenesis of cervical spondylosis have been widely discussed in the literature. The major emphasis in previous work has been on the narrowness of the spinal canal [1, 2], the range of movement of the spinal cord within the canal [3], and the constant friction of the cord against the spur [4]. The dentate ligament [5] and the hypertrophied ligamentum flavum [6] have been considered as etiologic factors. Ischemia of the gray 
matter and long tracts resulting from pressure on the anterior spinal artery [7] or the radicular arteries [8] has also been advanced by many authors as a cause of myelopathy. The arterial blood supply to the spinal cord comprises a centrifugal "central" system supplying the gray matter and corticospinal tracts and a centripetal "peripheral" system supplying the dorsal columns and periphery of the cord. Constant direct pressure on the cord probably causes flattening of the ventral surface and eventual central infolding (fig. 1A). Slowly progressive ischemia in the region of the anterior spinal artery would then produce degeneration of the anterior horns and the corticospinal tracts. This degeneration could account for the wasting of the anterolateral and posterolateral funiculi observed on CT in the late stages of spondylosis. Mair and Druckman [7] and Wilkinson [9] demonstrated these histologic changes in autopsies of patients with spondylotic myelopathy; Hukuda and Wilson [10] and Shimomura et al. [11] produced similar changes in experimental ischemia of the canine cord. The "notching" deformity in the posterior funiculus seen in patients with astereognosis of the hands (fig. 1C) probably reflects ascending degeneration in the cuneate fasciculus and/or atrophy at the entry zone of the dorsal roots into the cord.

Major histopathologic changes in the spinal cord in motor neuron disease include loss of motor cells and definite fiber loss with degeneration of the corticospinal tracts and preservation of the dorsal columns [12]. There is loss of myelin in the ventrolateral columns, most conspicuously in the direct and crossed pyramidal tracts, secondary to degeneration of Betz cells in the motor cortex. More prominent surface distortion of the spinal cord is prevented by reactive gliosis occurring within degenerated corticospinal tracts [13]. In our study, three patients who presented with a "monomelic" distal muscular atrophy of one hand, as described by Sobue et al. [14], unexpectedly showed an ipsilateral flattening of the anterolateral funiculus (fig. 2A), probably reflecting an underlying degeneration of the anterior horn cells. The combined anterior and posterior flattening in amyotrophic lateral sclerosis (fig. 2B) is due to degeneration of both the anterior horn cells and corticospinal tracts. None of the 11 patients with motor neuron disease had central flattening or infolding of the cord. The latter finding appears to be characteristic of spondylotic myelopathy. Metrizamide CT, if extended cephalad to include the brainstem, can show atrophy of the pyramidal eminences in amyotrophic lateral sclerosis (fig. 2C) or nonspecific atrophy in demyelinating disease.

Fog [15] studied the topographic distribution of demyelinating plaques in the spinal cord. They typically occur in the center of each posterior column, in the center and posterior two-thirds of the lateral columns, and in the anterior columns symmetrically on both sides of the anterior median fissure. Hughes [16] described a macroscopically generally atrophied spinal cord in multiple sclerosis with no segmental predilections. In the seven patients in our series with clinically definite multiple sclerosis, we found nonspecific atrophic changes of the spinal cord, including a small cord which occasionally had a wide ventral fissure and exaggerated surface features (fig. 3A). Only one patient with a 10 year history of the disease and left-sided hyperpathia from C2 to T2 had ipsilateral focal hemiatrophy (fig. 3B), in addition to diffuse atrophic changes. This focal deformity probably reflects a large demyelinating plaque, the location of which correlates with the patient's sensory symptoms. There was evidence of diffuse atrophy of the brainstem in three of the patients with multiple sclerosis. The atrophy can probably be attributed to plaques of focal demyelination. The diffusely small cord observed in our patients with multiple sclerosis probably reflects descending degeneration secondary to focal brainstem and/or cerebral involvement.
Metrizamide CT is far superior to routine myelography in the evaluation of spinal cord atrophy. In spondylotic myelopathy, there seems to be a wide spectrum of atrophic cord deformity, with central infolding as one of the most characteristic findings. Motor neuron disease is also best evaluated with metrizamide CT, whereby isolated flattening of the anterior funiculi or combined flattening of the anterior and posterolateral funiculi of the spinal cord are demonstrated, depending on whether there is degeneration of the lower or upper motor neurons or both. More than half the patients presenting with spastic paraparesis and definite multiple sclerosis had nonspecific atrophy of the spinal cord. Evaluation of the brainstem with metrizamide CT has proven to be of crucial importance in evaluating pyramidal degeneration in cases of amyotrophic lateral sclerosis or brainstem atrophy in multiple sclerosis.

\section{REFERENCES}

1. Payne EE, Spillane JD. The cervical spine. An anatomicopathological study of 70 specimens (using a special technique) with particular reference to the problem of cervical spondylosis. Brain 1957;80:571-596

2. Nurick S. The pathogenesis of the spinal cord disorder associated with cervical spondylosis. Brain 1972;95:87-100

3. Adams CBT, Logue V. Studies in cervical spondylotic myelopathy. II. The movement and contour of the spine in relation to the neurologic complications of cervical spondylosis. Brain 1971;84:569-586

4. Allen KL. Neuropathies caused by bony spurs in the cervical spine with special reference to surgical treatment. J Neurol Neurosurg Psychiatry 1952;15:20-36

5. Kahn EA. The role of the dentate ligaments in spinal cord compression and the syndrome of lateral sclerosis. I Neurosurg 1947; 4:191-199

6. Stoltman HF, Blackwood W. Role of ligaments flava in pathogenesis of myelopathy in cervical spondylosis. Brain $1964 ; 86: 45-50$

7. Mair WGP, Druckman R. The pathology of spinal cord lesions and their relation to the clinical features in protrusion of cervical intervertebral discs. Brain 1953;76:70-91

8. Frykholm R. Cervical nerve root compression resulting from disc degeneration and root sleeve fibrosis. Acta Chir Scand [Suppl] 1951;160:9-33

9. Wilkinson $M$. The morbid anatomy of cervical spondylosis and myelopathy. Brain 1960;83:589-617

10. Hukuda S, Wilson CB. Experimental cervical myelopathy: Effects of compression and ischemia on the canine cervical cord. J Neurosurg 1972;37:631-652

11. Shimomura $\mathrm{Y}$, Hukuda $\mathrm{S}$, Mizuno $\mathrm{S}$. Experimental study of ischemic damage to the cervical spinal cord. I Neurosurg 1968;28:565-581

12. Brownell B, Oppenheimer DR, Hughes JT. The central nervous system in motor neuron disease. J Neurol Neurosurg Psychiatry 1970;33:338-357

13. Lawyer T, Netsky M. Amyotrophic lateral sclerosis. Arch Neurol 1953;69:171-192

14. Sobue I, Saito N, lida M, Ando K. Juvenile type of distal and segmental muscular atrophy of upper extremities. Ann Neurol 1978;3:429-432

15. Fog T. Topographic distribution of plaques in spinal cord in multiple sclerosis. Arch Neurol 1950;63:382-414

16. Hughes JT. Major problems in pathology. Vol 6. Pathology of the spinal cord. Philadelphia: Saunders, 1978 